\title{
Tin-Free Three-Component Coupling Reaction of Aryl Halides, Norbornadiene (or Norbornene), and Alkynols Using a Palladium Catalyst
}

\author{
Cheol-Kyu Choi, Jin-Who Hong, ${ }^{\dagger, *}$ Ikuyoshi Tomita, ${ }^{\ddagger}$ and Takeshi Endo \\ Institute of Photonics and Surface Treatment. O-sys Co., 985-18, Jangduk-dong, Gwangsan-gu, Kw'angin 506-251, Korea \\ ${ }^{\dagger}$ Department of Polmer Science and Engineering, Chostm Lniversitw, 375, Sustk-dong, Dong-gu, Ki'angit 501-759, Korea

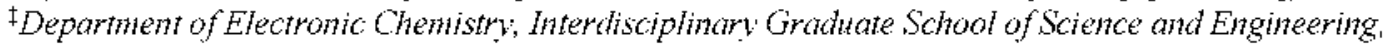 \\ Tokvo Institute of Technologv, Nagatua-cho 4259, Aidori-ku, Yokohama 226-8502, Japan \\ $\$$ Department of Polymer Science and Engineering Faculty of Engineering Famagata Lniversity $4-3-16$ Jonan, \\ Ionezawa, Iamagata 992-8510, Japan \\ Received June 18, 2001
}

\begin{abstract}
Good-to-excellent yields of 2.3-Disubstituted norbornenes (or norbornanes) were obtained using a $\mathrm{Pd} / \mathrm{Cu}$ catalyzed three-component ternary coupling reaction of aryl halides. norbornadiene (or norbornene). and alkynols in toluene at $100{ }^{\circ} \mathrm{C}$ in the presence of $5.5 \mathrm{M} \mathrm{NaOH}$ as a base and benzyltriethylammonium chloride as a phase transfer catalyst. The results of experiments using various aromatic halides suggest that the ternary coupling reaction is promoted by bromide.
\end{abstract}

Keywords : Aryl halides. Norbomene, Alkynols. Palladium-catalyst, Three-conponent reaction.

\section{Introduction}

Transition metal-catalyzed multi-component coupling reactions are important in the single step construction of complex molecules. ${ }^{1-7}$ They are also potentially of importance in the development of a new precision polycondensation technique in which more than three kinds of building block can be sequentially incoperated into polymer chains. ${ }^{8}$

In general, the reported tenlary coupling systems consist of aromatic halides (or vinyl halides). nucleophiles. and unsaturated systems such as allenes, carbon monoxide. and olefins. To ensure that the objective reaction is selective. great efforts have been made to axoid the direct crosscoupling of organic halides with nucleophiles. For example. Kosugi et $a l^{y-13}$ have reported that the reaction of aryl halides, norbornadiene (or norbornene), and organostanuanes in the presence of a palladium complex provides an analogous overall transformation. Although acetylenes containing heteroatoms at their propargylic positions are also suitable for achieving the ternary coupling reaction. ${ }^{14-16}$ the use of terminal acetylenes might give rise to binary coupling products of aryl halides with the acety lenes in addition to the tentary coupling products (Scheme 1). Owing to the toxicity and rather high price of organotin compounds, ${ }^{17-18}$ it is of

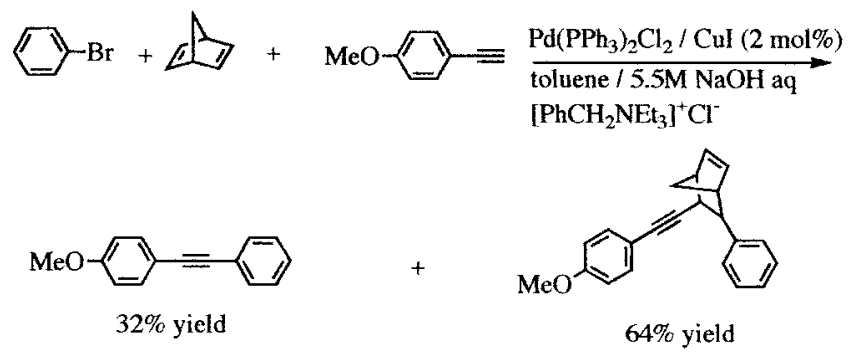

Scheme 1 practical importance to find an altemative candidate that could be employed in temary coupling involving organic halides and norbornene.

On the basis of the fact that alkynols serve as equivalence of terminal acetylenes. ${ }^{19}$ we report here teclunique for achieving three-component coupling of aromatic halides and norbonadiene using alkynols as one of the components (Scheme 2).

\section{Results and Discussion}

The three-component coupling reaction using bromobenzene (1A). 4-methoxyphenyl-2-methyl-3-butyn-2-ol (4a), and norbomadiene (2) was carried out at $100^{\circ} \dot{C}$ for 2 days in toluene/5.5 M NaOH in the presence of benzyltriethylammonium chloride and $\mathrm{Pd}(\mathrm{PPl} 3) 2 \mathrm{Cl} 2 / \mathrm{CuI}$ catalyst $(2 \%$
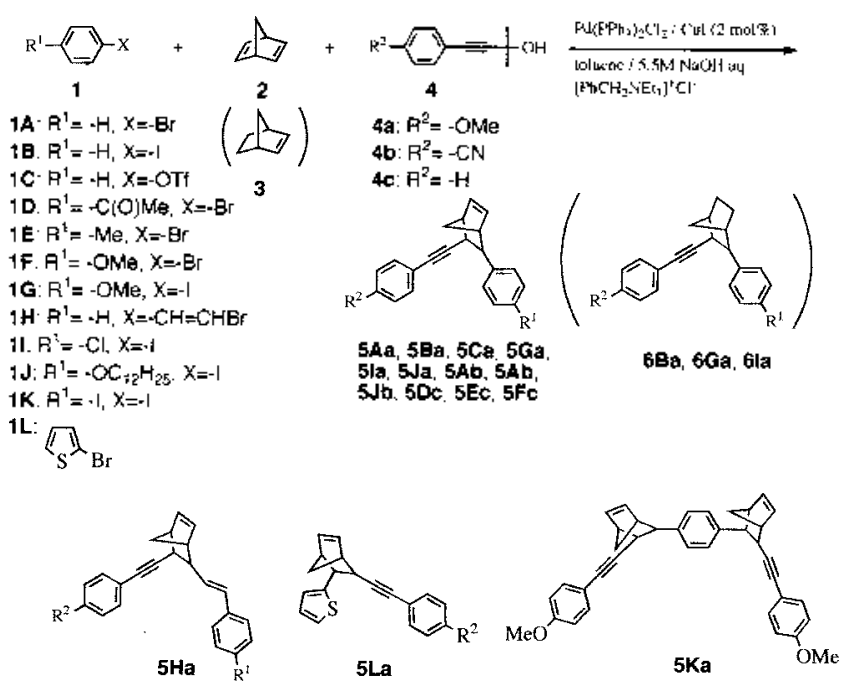

Scheme 2 


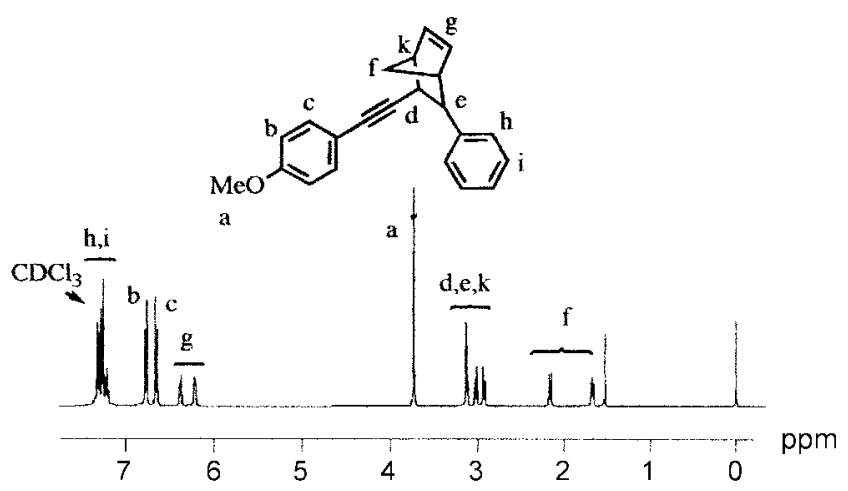

Figure 1. ${ }^{1} \mathrm{H}-\mathrm{NMR}$ spectrum of the 5 Aa.
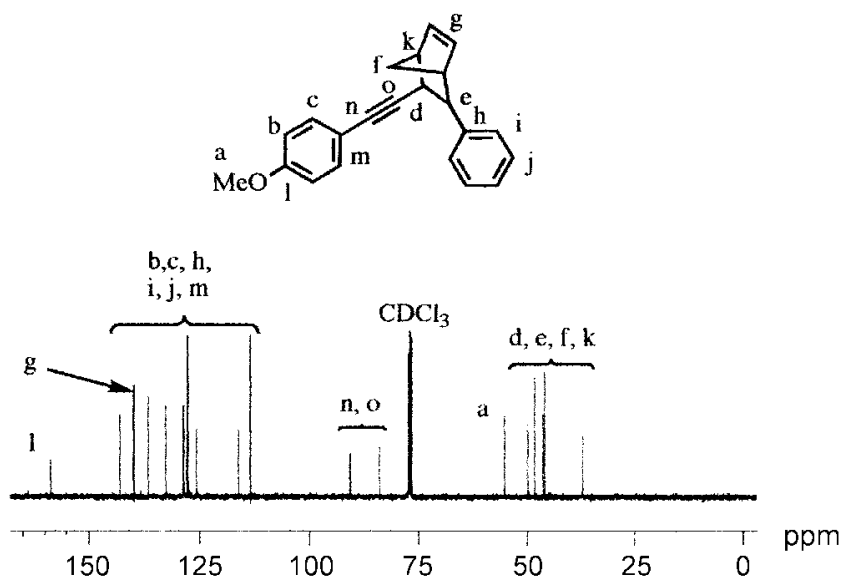

Figure 2. ${ }^{13} \mathrm{C}-\mathrm{NMR}$ spectrum of the $\mathbf{5 A a}$.

molality).

A $94 \%$ yield of 2.3 -disubstituted norbornene (5Aa) was obtained (Table 1. run 1). The proposed structure of $\mathbf{5 A a}$ was confirmed objectively by ${ }^{1} \mathrm{H}-\mathrm{NMR} .{ }^{13} \mathrm{C}-\mathrm{NMR}$, IR spectrometry. and elemental analysis. For example. ${ }^{1} \mathrm{H}-\mathrm{NMR}$ spectrum strongly supported that 5Aa consists of building blocks originated from the three kinds of components (Figure 1). That is. peaks originated from three different components were observed at $6.22,6.37$ (the double bond in norbonene moieties). 6.65. 6.67 (phenylene originated from alkynol).
Table 1. Palladium-catalyzed temary coupling reaction of 1 A, 2. and $3 \mathbf{a}$ in the presence of bases ${ }^{a}$

\begin{tabular}{ccc}
\hline Run & Base & Yield $/ \% \%^{{ }^{t}}$ \\
\hline 1 & $\mathrm{NaOH}$ & 94 \\
2 & $\mathrm{KOH}$ & 91 \\
3 & $\mathrm{~K}_{2} \mathrm{CO}_{3}$ & 62 \\
4 & $\mathrm{CH}_{3} \mathrm{CO}_{3} \mathrm{~K}$ & 14 \\
5 & $\mathrm{CH}_{3} \mathrm{CO}_{2} \mathrm{Na}$ & 5 \\
\hline
\end{tabular}

Reaction conditions: 1A $(0.5 \mathrm{mmol}) .2(10 \mathrm{mmol}) .3 \mathrm{a}(0.5 \mathrm{mmol})$ $\mathrm{PdCl}_{3}\left(\mathrm{PPh}_{3}\right)_{2}(0.0 \mathrm{~lm} \mathrm{~mol})$. $\mathrm{CuI}(0.01 \mathrm{mmol}) .5 .5 \mathrm{M}$ aq base $(0.3 \mathrm{~mL})$. benzyltriethylammonium chloride $(0.015 \mathrm{mmol})$. toluene $(2 \mathrm{~mL})$, at 100 ${ }^{\mathrm{C}} \mathrm{C}$ for 2 days ${ }^{b} \mathrm{Isolated}$ vields by column chromatography (SiO. ${ }^{-}$ hexane : ethyl acetate $=4: 1$ ).

and $7.27 \mathrm{ppm}$ (phenyl from bromobenzene), respectively:

In the ${ }^{13} \mathrm{C}$-NMR spectrum. the signals for three building blocks were also observed at 83.9.93.7 (the carbon atoms of acetylene moieties). 139.8 (the carbon atoms of the double bond in norbomene). and $55.1 \mathrm{ppm}$ ( the carbon atom of methoxy), respectively (Figure 2). The structure of $\mathbf{5 A a}$ was identified as the cis, exo form on the basis of observations on the structure of the products obtained using the method of Kosugi et $a l^{9.13}$ and Chiusoli et $a l^{3-3}$

The rate of the ternary coupling reaction largely depends on the inorganic bases employed. Both $\mathrm{NaOH}$ and $\mathrm{KOH}$ exhibiting strong basicity - are more suitable (Table 1 . runs 1 and 2) than bases exhibiting weaker basicity (Table 1. runs 3-5). Thus. $\mathrm{NaOH}$ was used for the present study. The effect of $\mathrm{NaOH}$ on the temary coupling reaction was investigated using bromobenzene (1A) and iodobenzene (1B) (Figures 3 and 4 ). The ternary coupling reaction did not take place in the absence of $\mathrm{NaOH}$. but the prence of at least an equimolar amount of $\mathrm{NaOH}$ resulted in a smooth reaction. The addition of excess $\mathrm{NaOH}$ accelerated the reaction. although the yield of the product did not change significantly. From the conversion of $1 \mathrm{~A}$ and $\mathbf{1 B}$ monitored by gas chromatograply (GC), it was confinmed that the ternary coupling reaction proceeded smoothly to reach $100 \%$ conversion within $8 \mathrm{~h}$. The results of the ternary coupling reactions of bromobenzene. iodobenzene. and phenyl triflate (1A, 1B. and 1C, (a)

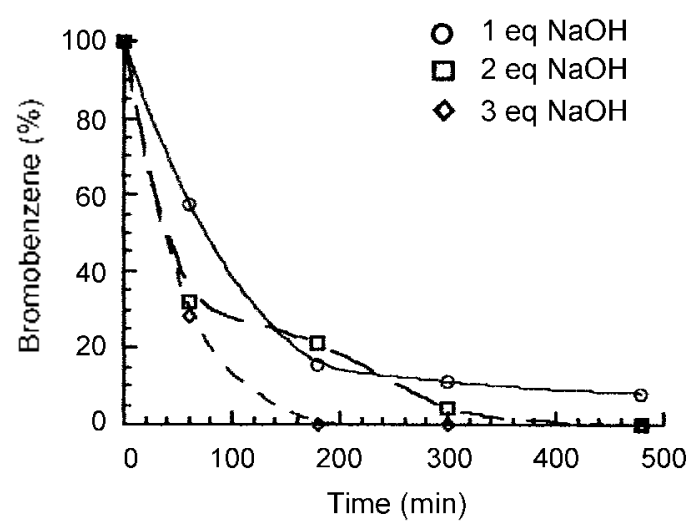

(b)

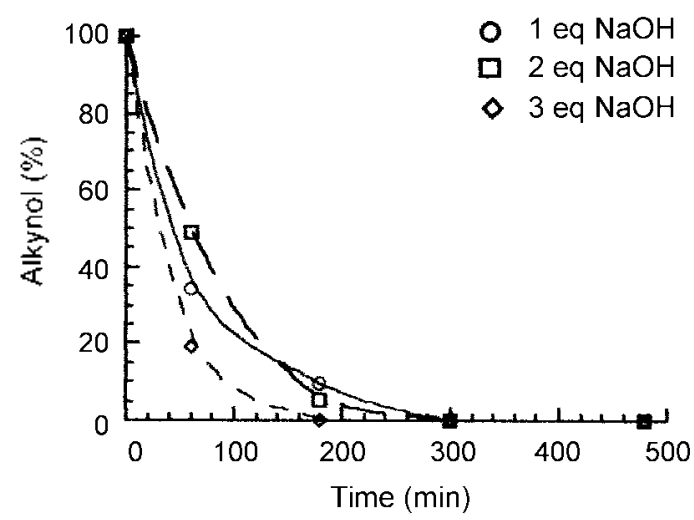

Figure 3. Time-conversion curves of bromobenzene (a) and alkynols (b) in the temary coupling reaction. 

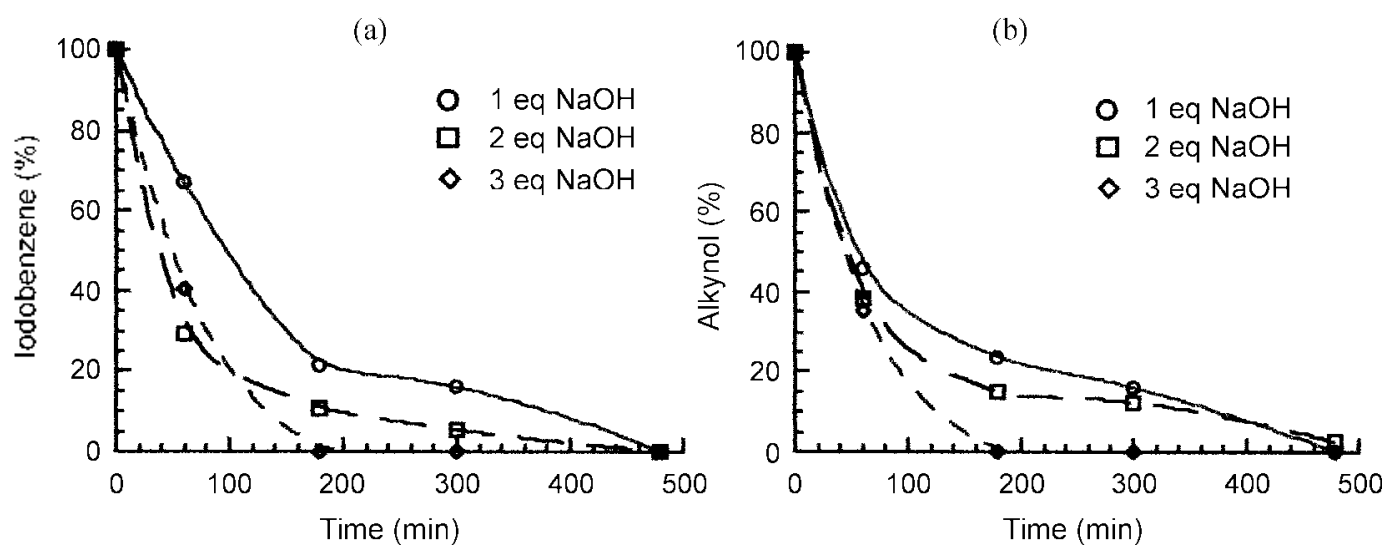

Figure 4. Tune-conversion curves of iodobenzene $\mathrm{c}$ and alkynols (b) in the ternary coupling reaction.

respectively). 2. and $4 a$ are summarized in Table 2 (runs l3). Fron 1B. a $73 \%$ yield of the coupling product was obtained after the reaction had proceeded for 2 days. In the case of $1 \mathrm{C}$. the ternary coupling reaction at 100 ( $\mathrm{C}$ for 2 days produced a low product yield ( $41 \%$ ). although its conversion was complete within the reaction after $1 \mathrm{~h}$, which night be due to the competitive hydrolysis of $1 \mathrm{C}$. In fact. when two equivalent anounts of $\mathbf{1 C}$ were used under the same reaction conditions, the yield of the product increased to $79 \%$ (Table 2, ruil 4).

From the experiments using aryl halides bearing substitutents on the para position in the ternary coupling reaction with 2 and alkynols ( $4 a$ or 4 c), those having electrondonating substitutents (1A and 1E-1G) gave excellent yields of the desired products (Table 2 . runs $1,5,10.12$. and 13). while that having an electron-withdrawing group gave a slightly lower yield of the product (Table 2, run 6). Likewise. alkynols having electron-withdrawing groups reduced

Table 2. Palladium-catalyzed ternary coupling reaction of aryl halides (1A-1G and 1I) 2 , and alkynols $(4 \mathrm{a}-4 \mathrm{c})^{a}$

\begin{tabular}{|c|c|c|c|}
\hline Runn & Aryl Halide & AlkTnol & Yield $(\%)^{b}$ \\
\hline 1 & $1 \mathrm{~A}$ & $4 a$ & 94 \\
\hline 2 & 1B & ta & 73 \\
\hline 3 & $1 \mathrm{C}$ & $4 a$ & 41 \\
\hline $4 c$ & $1 \mathrm{C}$ & ta & 79 \\
\hline 5 & $1 G$ & $4 a$ & 92 \\
\hline 6 & $1 I$ & ta & 63 \\
\hline 7 & 1B & $4 b$ & 54 \\
\hline $8^{i 7}$ & 1B & $4 b$ & 61 \\
\hline 9 & $1 \mathrm{~A}$ & $4 b$ & 65 \\
\hline 10 & $1 \mathrm{~A}$ & $4 c$ & 93 \\
\hline 11 & $1 D$ & $4 c$ & 73 \\
\hline 12 & $1 E$ & $4 c$ & 99 \\
\hline 13 & $1 F$ & $4 c$ & 98 \\
\hline
\end{tabular}

"Reaction conditions: 1.A-1F $(0.5 \mathrm{mmol})$, 4a-te $(0.5 \mathrm{mmol}), 2$ (10 mmol). $\mathrm{Pd}\left(\mathrm{PPd}_{2}\right)_{2} \mathrm{Cl} l_{2}(0.01 \mathrm{mmol})$. $\mathrm{CuI}(0.01 \mathrm{mmol}) .5 .5 \mathrm{~N}$ aq $\mathrm{NaOH}$ $60.2 \mathrm{~mL}$ ) benzyltriethylammonium chloride $(0.015 \mathrm{mmol})$, toluene $(2$ $\mathrm{mL}$ ), at $100^{\circ} \mathrm{C}$ for 2 davs. "Isolated vields by column chromatography ( $\mathrm{SiO}_{2}$ m-hexane:ethyl acetate $\left.=4: 1\right)$. 'Phenvl triflate $(1 \mathrm{mmol})$ was used. "Sodium bromide $(0.5 \mathrm{mmol})$ was used. the yield of the products (Table 2. runs 7 and 9). It is noteworthy that the addition of $\mathrm{NaBr}$ to the reaction mixture led to a substantial increase in the yield of the products and the conversion of the starting materials (Figure 3 and Table 2. run 8). Although the role of $\mathrm{NaBr}$ remains unclear. one possible explanation is that the added bromide displace the palladium ligand. ${ }^{20}$

The palladium-catalyzed temary coupling reaction was investigated using various aryl halides. norbonadiene (or norbonene). and alkynols. The results are summarized in Table 3. Aryl halides such as (E)- $\beta$-bromostyrene, 2-bromothiophene, para-substituted iodobenzene. and 1,4-diiodobenzene produced excellent yields of the corresponding temary coupling products (Table 3, runs 1-4). As an olefin component, norbonene (3) also produced excellent yields of the corresponding temary coupling products (Table 3, runs 5-7).

On the basis of the reported mechanism for the palladiumcatalyzed coupling reaction, ${ }^{2,3,113}$ we propose that the present reaction pathway was as shown in Scheme 3. That is. the catalytic cycle may consist of four distinct stages: (a) oxidative addition of the aryl halide (B) to $\mathrm{Pd}(0)$ (A). (b) insertion of norbornadiene (D) to the aryl-Pd moieties $(C)$. (c) formation of the complex $(\mathbf{H})$ via transmetallation with a copper acetylide $(\mathbf{G})$ generated by the reaction of alkynol (F) with copper (I) in the presence of base. and (d) reductive

Table 3. Palladium-catalyzed coupling reaction of various aryl halides (1B, 1G-1L), 2 (or 3), and various alkynols $(4 \mathbf{a}-4 \mathbf{b})^{n}$

\begin{tabular}{ccccc}
\hline Run & Aryl Halide & Olefin & Alkynol & Yield $(\%)^{b}$ \\
\hline l & 1H & $\mathbf{2}$ & $\mathbf{4 a}$ & 93 \\
2 & $1 \mathbf{J}$ & $\mathbf{2}$ & $\mathbf{4 b}$ & 92 \\
3 & $1 \mathbf{K}$ & $\mathbf{2}$ & $\mathbf{4 a}$ & 81 \\
4 & $1 \mathbf{L}$ & $\mathbf{2}$ & $\mathbf{4 a}$ & 88 \\
5 & $1 \mathbf{B}$ & $\mathbf{3}$ & $\mathbf{4 a}$ & 97 \\
6 & $1 \mathbf{G}$ & $\mathbf{3}$ & $\mathbf{4 a}$ & 98 \\
7 & $1 \mathbf{I}$ & $\mathbf{3}$ & $\mathbf{4 a}$ & 85 \\
\hline
\end{tabular}

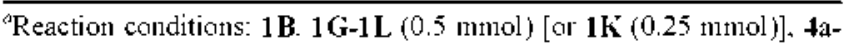
4b $(0.5 \mathrm{mmal})$ ). 2 (or 3$)$ ( $10 \mathrm{mmal}$ ). PdCl $2\left(\mathrm{PPh}_{3}\right) 2(0.01 \mathrm{mmal})$. CuI $(0.01$ mmol). $5.5 \mathrm{M}$ ac $\mathrm{NaOH}(0.2 \mathrm{~mL})$. benzyltriethylammonium chloride $(0.015 \mathrm{mmol})$. toluene $(2 \mathrm{~mL})$ at $1000^{\circ} \mathrm{C}$ for 2 days ${ }^{b} \mathrm{Isolated}$ yields by chromatography $\left(\mathrm{SiO}_{2}, n\right.$-hexane $:$ ethyl acetate $\left.=4: 1\right)$. 
elimination of complex (H) produces the 2.3-disubstituted norbornene product (I) with regeneration of the $\operatorname{Pd}(0)(A)$ species.

In summary, a convenient method for a tin-free threecomponent coupling reaction of aryl halides. norbornadiene (or norbornene) with alhynols has been successfully developed to provide good-to-excellent yields of the desired 2,3disubstituted norbonnenes (or norbonanes).

\section{Experimental Methods}

General Considerations. Nuclear magnetic resonance (NMR) spectra were recorded in $\mathrm{CDCl}_{3}$ on a JNM-EX400 spectrometer ( ${ }^{1} \mathrm{H}$ NMR: $400 \mathrm{MHz}$ and ${ }^{13} \mathrm{C} \mathrm{NMR}, 100 \mathrm{MHz}$ ). IR spectra were obtained on a JASCO FT/IR-5300 spectrometer. Gas chromatography (GC) analyses were performed out on a Shimadzu GC-14B equipment. Mass spectra were obtained by EI method or the FAB method using a Hitachi $\mathrm{M}-80$ or a JEOL JMS-SX102 mass spectrometer. Toluene was dried and deoxygenated by refluxing and distilling from sodium/benzophenone ketyl under nitrogen. Bromobenzene $(\mathbf{A})$, iodobenzene (1B), phenyl trifluoromethanesulfonate (1C), 4-bromotoluene (1E), 4-bromoanisole (1F). (E)- $\beta$ bromostyrene $(\mathbf{1 H})$, and 2-bromothiophene $(\mathbf{1 L})$ were purified by distillation. 4'-Bromoacetophenone (1D), 4-Iodoanisole (1G), 4-chloroiodobenzene (1I), and 1.4-diiodobezene $(\mathbf{1 K})$ were purified by recrystallization from $n$ hexane. 4-Dodecyloxyiodobenzene (1.J) was prepared by the reaction of 4-iodophenol with 12-bromododecane and was purified by recrystallization from $n$-hexane.

Norbonadiene (2) and norbonene (3) were dried over $\mathrm{CaH} 2$ and distilled under nitrogen. All the reactions were carried out in a degassed sealed tube

Synthesis of Alkynols. ${ }^{19}$ The typical procedure, for 4Methosyphenyl-2-methyl-3-butyn-2-ol (4a). was as follows. 4-Iodoanisole $(4.680 \mathrm{~g} .20 \mathrm{~mm}$ ol). palladium(II) chloride $(0.0469$ g. $0.4 \mathrm{munol})$ triphenylphosphine $(0.3148 \mathrm{~g} .1 .2$ $\mathrm{mmol})$, copper (I) iodide $(0.1524 \mathrm{~g}, 0.8 \mathrm{mmol}), 2-\mathrm{methyl}-3$ butyn-2-ol $(1.851 \mathrm{~g}, 22 \mathrm{mmol})$, diethylamine $(20 \mathrm{~mL})$. and THF $(40 \mathrm{~mL})$ were added to a $100-\mathrm{mL}$ two-necked. round bottom flask equipped with a reflux condenser. magnetic stirrer chip. and nitrogen-gas inlet. The mixture was stirred at ambient temperature for $5 \mathrm{~h}$ under nitrogen. and then the solvent was evaporated under vacuun. After the addition of water $(200 \mathrm{~mL})$ and diethyl ether $(100 \mathrm{~mL})$, the organic layer was separated and dried over $\mathrm{MgSO}_{4}$. After removal of the solvent in victo, the product was recrystallized from toluene to produce a $98 \%$ yield of white powders $(3.73 \mathrm{~g}$. $19.6 \mathrm{mmol})$. ${ }^{\mathrm{l}} \mathrm{H}-\mathrm{NMR}\left(400 \mathrm{MHz} . \delta\right.$, ppm) $1.64\left(\mathrm{CH}_{3}\right.$. s. $\left.6 \mathrm{H}\right)$. $2.09(-\mathrm{OH}$. s. $\mathrm{lH}) .3 .83\left(-\mathrm{OCH}_{3}, \mathrm{~s}, 3 \mathrm{H}\right), 6.86\left(-\mathrm{C}_{6} \mathrm{H}_{4}, \mathrm{~d}, J=\right.$ $4.4 \mathrm{~Hz} .2 \mathrm{H}) .7 .38\left(-\mathrm{C}_{6} \mathrm{H}_{4} \cdot \mathrm{d} . J=4.4 \mathrm{~Hz} .2 \mathrm{H}\right),{ }^{13} \mathrm{C}-\mathrm{NMR}(100$ $\mathrm{MHz}, \delta$, ppm $) 31.2\left(-\mathrm{C}\left(\mathrm{CH}_{3}\right)_{2} \mathrm{OH}\right) .54 .9\left(-\mathrm{C}_{6} \mathrm{H}_{4} \mathrm{OCH}_{3}-\right) .65 .3$ $\left(-\underline{\mathrm{C}}\left(\mathrm{CH}_{3}\right)_{2} \mathrm{OH}\right), 81.6 .92 .0 \quad(-\underline{\mathrm{C}} \equiv \underline{\mathrm{C}}-) .113 .5 .114 .4,132.7$. 159.2: IR (KBr) 3354 (-OH). 3057. 3034. 2982. 2934. 2870. $2231(-\mathrm{C} \equiv \mathrm{C}-), 1952,1880.1807,1720,1672,1599.1574$. $1491,1444,1363,1275,1163,1070,1026,1001,962.906$, $842.808,756.692,667 \mathrm{~cm}^{-1}$
For 4-cyanophenyl-2-methyl-3-butyn-2-ol (4b) the procedure was as follows: from 4-bromobenzonitrile ( $1.82 \mathrm{~g} .10 .0$ mmol) and 2-methyl-3-butyn-2-ol (925 mg. $11.0 \mathrm{mmol}$ ); yield $86 \%(1.59$ g. $8.60 \mathrm{mmol})$ : ${ }^{1} \mathrm{H}-\mathrm{NMR}(400 \mathrm{MHz}, \delta$, ppm) $1.62\left(\mathrm{CH}_{3}\right.$. s. $\left.6 \mathrm{H}\right), 2.20(-\mathrm{OH}, \mathrm{s}, \mathrm{lH}) .7 .48\left(-\mathrm{C}_{6} \mathrm{H}_{4} \cdot \mathrm{d}, J\right.$ $=8.8 \mathrm{~Hz}, 2 \mathrm{H}), 7.59\left(-\mathrm{C}_{6} \mathrm{H}_{4}-\mathrm{d}, J=8.8 \mathrm{~Hz} .2 \mathrm{H}\right) ;{ }^{13} \mathrm{C}-\mathrm{NMR}$ (100 MHz. $\delta$. ppm) $31.2\left(-\mathrm{C}\left(\mathrm{CH}_{3}\right)_{2} \mathrm{OH}\right) .65 .5\left(-\mathrm{C}\left(\mathrm{CH}_{3}\right)_{2} \mathrm{OH}\right)$, $80.5 .98 .2(-\mathrm{C}=\mathrm{C}-\mathrm{-}), 111.5,118.4(-\mathrm{C}=\mathrm{N}) .127 .7 .132 .1$; $\mathrm{IR}$ $(\mathrm{KBr}) 3410(-\mathrm{OH}), 3045.2980,2932.2876,2797.2241$ $(-\mathrm{C}=\mathrm{N}), 2226(-\mathrm{C}=\mathrm{C}-), 1927,1803,1676,1601,1560,1498$, 1456. 1402, 1369, 1273. 1188. 1165, 1107. 1014. 962, 906, $839.723,625.561,486 \mathrm{~cm}^{-1}$.

4-Phenyl-2-methyl-3-butyn-2-ol (4c): from bromobenze $(1.5702 \mathrm{~g} .10 \mathrm{mmol})$ and 2-methyl-3-butyn-2-ol $(0.9250 \mathrm{~g}$. $11 \mathrm{mmol}$ ); Yield $91 \%$ (1.4580 g. $9.1 \mathrm{mmol})$; $\mathrm{H}-\mathrm{NMR}$ ( 400 $\mathrm{MHz}, \delta . \mathrm{ppm}) 1.61\left(\mathrm{CH}_{3}\right.$. s. $\left.6 \mathrm{H}\right), 2.62(-\mathrm{OH}, \mathrm{s} . \mathrm{lH}) .7 .28$ $\left(-\mathrm{C}_{6} \mathrm{H}_{4} \cdot . \mathrm{d}, J=2.4 \mathrm{~Hz} .3 \mathrm{H}\right) .7 .41\left(-\mathrm{C}_{6} \mathrm{H}_{4} \cdot \mathrm{m}, 2 \mathrm{H}\right) .{ }^{13} \mathrm{C}-\mathrm{NMR}$ (100 MHz. $\delta$ ppm) $31.3\left(-\mathrm{C}\left(\mathrm{CH}_{3}\right)_{2} \mathrm{OH}\right) \cdot 65.4\left(-\mathrm{C}\left(\mathrm{CH}_{3}\right)_{2} \mathrm{OH}\right)$, 81.9. $93.8(-\mathrm{C}=\mathrm{C}-), 122.6,128.1 .131 .9 .132 .1 ; \mathrm{IR}(\mathrm{KBr})$ $3435(-\mathrm{OH}) .3119,3074.3022$. 2988. 2935, 2841. 2532 . 2226 (-C=C-), 2050. 2008. 1896, 1772. 1649, 1606, 1570. 1508. 1454. 1440. 1363. 1304. 1286. 1248. 1168. 1107, $1030.962 \mathrm{~cm}^{-1}$

Temary Coupling Reaction of Norbomadiene, Aromatic Halides. and Alkynols (General Procedure). A tube containing a mixture of norbonadiene $(10 \mathrm{mmol})$. aromatic halide $(0.5 \mathrm{mmol})$, alkynol $(0.5 \mathrm{mmol}), \mathrm{Pd}\left(\mathrm{PPl}_{2}\right)_{2} \mathrm{Cl}_{2}(0.01 \mathrm{mmol})$, triphenylphosphine $(0.055 \mathrm{mmol}), \mathrm{Cul}(0.01 \mathrm{mmol})$. benzyltriethyl ammonium chloride (0.015 nmol). aqueous $\mathrm{NaOH}$ $(5.5 \mathrm{~N} .0 .2 \mathrm{~mL})$. and toluene ( $2 \mathrm{~mL}$ ) was sealed in vacuo, immeresed in an oil bath. and heated at $100^{\circ} \mathrm{C}$ for 2 days. Products were isolated by silica gel column chromatography ( $n$-hexane:ethyl acetate $=4: 1$ as eluent).

Products. cis, exo-2-Phenyl-3-(4-methoxyphenylethynyl)bicyclo-[2,2.1]heptene (5Aa): ${ }^{1} \mathrm{H}$ NMR $\left(400 \mathrm{MHz}, \mathrm{CDCl}_{3}\right)$ $\delta \mathrm{I} .68\left(>\mathrm{CH}_{2}\right.$ (7). d. $\left.J=8.8 \mathrm{~Hz}, \mathrm{lH}\right), 2.17\left(>\mathrm{CH}_{2}\right.$ (7). d. $J=$ $8.8 \mathrm{~Hz}, 1 \mathrm{H}$ ). 2.94 (d. $J=8.8 \mathrm{~Hz}, 1 \mathrm{H}) .3 .03$ (d. $J=8.8 \mathrm{~Hz}$, $1 \mathrm{H}) .3 .14(\mathrm{~s}, 2 \mathrm{H}) .3 .75\left(-\mathrm{OCH}_{2} . \mathrm{s}, 3 \mathrm{H}\right), 6.24(-\mathrm{CH}=\mathrm{CH}-(5$. 6). s. $\mathrm{lH}), 6.39(-\mathrm{CH}=\mathrm{CH}-(5,6) . \mathrm{s}, \mathrm{lH}) .6 .65\left(-\mathrm{C}_{6} \mathrm{H}_{4} \cdot \mathrm{d}, J=\right.$ $8.8 \mathrm{~Hz}, 2 \mathrm{H}), 6.77\left(-\mathrm{C}_{6} \mathrm{H}_{4}=\right.$ d. $\left.J=8.8 \mathrm{~Hz}, 2 \mathrm{H}\right), 7.23-7.35$ $\left(-\mathrm{C}_{6} \mathrm{H}_{5} . \mathrm{m} .5 \mathrm{H}\right) \mathrm{ppm:}{ }^{13} \mathrm{C}$ NMR $\left(100 \mathrm{MHz} . \mathrm{CDCl}_{3}\right) \delta 37.2$, 45.9. 48.3, 49.8, 55.2 (- $\left.\mathrm{C}_{6} \mathrm{H}_{4} \mathrm{OC}_{3}-\right), 84.0,90.7$ (-C= $\left.=\underline{\mathrm{C}}-\right)$. 113.5, 116.1. 125.7. 127.7. 128.7. 132.6, 136.6, 143.0, 158.8 ppm: IR (KBr) 3053. 2970. 2872, 2837. 2222 (-C=C-). 1890 , 1606. $1568(-\mathrm{CH}=\mathrm{CH} \cdot(5,6)), 1510,1454.1442 .1327$. 1286. 1249. 1172. 1105. 1028. 912 . 854, 829. 808, 756.731. 715. 700. 655. 534, $\mathrm{cm}^{-1}$; MS (EI) $\mathrm{m} / \mathrm{z} 300.1414(\mathrm{M}+)$; Anal. Calcd for $\mathrm{C} 22 \mathrm{H} 20 \mathrm{O} ; \mathrm{C}, 87.96$; H. 6.71; O, 5.33 , Found: C, 87.52: $\mathrm{H}, 6.72$.

cis. exo-2-(4-Methoxy)phenyl-3-(4-methoxyphenylethynyl)bicyclo[2.2,1]-heptene (5Ga): ${ }^{~} \mathrm{H}$ NMR $\left(400 \mathrm{MHz}, \mathrm{CDCl}_{3}\right)$ $\delta 1.66\left(>\mathrm{CH}_{2}\right.$ (7). d. $\left.J=8.8 \mathrm{~Hz}, 1 \mathrm{H}\right), 2.15\left(>\mathrm{CH}_{2}(7)\right.$. d. $J=$ $8.8 \mathrm{~Hz}, \mathrm{LH}$ ). 2.90 (d. $J=8.8 \mathrm{~Hz}, 1 \mathrm{H}$ ). 2.97 (d. $J=8.8 \mathrm{~Hz}$, IH). $3.08(\mathrm{~d}, J=17.6 \mathrm{~Hz}, 2 \mathrm{H}), 3.73\left(-\mathrm{OCH}_{3}, \mathrm{~s} .3 \mathrm{H}\right) .3 .8 \mathrm{l}$ $\left(-\mathrm{OCH}_{2}\right.$. s. $\left.3 \mathrm{H}\right), 6.20(-\mathrm{CH}=\mathrm{CH} \cdot(5.6) . \mathrm{s}, 1 \mathrm{H}) .6 .37(-\mathrm{CH}$ $=\mathrm{CH}-(5,6), \mathrm{s}, \mathrm{lH}) .6 .67\left(-\mathrm{C}_{6} \mathrm{H}_{4}-\mathrm{d}, 2 \mathrm{H}\right) .6 .85\left(-\mathrm{C}_{6} \mathrm{H}_{4}-\mathrm{m}\right.$, $4 \mathrm{H}) .7 .20\left(-\mathrm{C}_{6} \mathrm{H}_{4^{-}}\right.$, d. $\left.2 \mathrm{H}\right) \mathrm{ppm}:{ }^{13} \mathrm{C}$ NMR $(100 \mathrm{MHz}$. 
$\left.\mathrm{CDCl}_{3}\right) \delta 37.7 .46 .2 .46 .9 .47 .9,50.8 .56 .2\left(-\mathrm{C}_{6} \mathrm{H}_{4} \mathrm{OCH}_{3}-\right)$. $84.6,91.9(-\mathrm{C}=\mathrm{C}-\mathrm{-}), 113.8 .116 .5,130.0,133.4 .136 .2 .137 .7$. $140.9,158.3,159.3 \mathrm{ppm}$; IR (KBr) 3061, 3024. 2957, 2912. $2870,2839.2224(-\mathrm{C}=\mathrm{C} \cdot) .1896 .1606,1570(-\mathrm{CH}=\mathrm{CH} \cdot(5$. 6)). 1508. 1466, 1452. 1331, 1290. 1246, 1174, 1109. 1076. $1039,910.835,785.744,723.698,663.619,538 \mathrm{~cm}^{-1}$; MS (EI) $\mathrm{m} / \mathrm{z} 330.1526(\mathrm{M}+)$ : Anal. Calcd for $\mathrm{C}_{33} \mathrm{H}_{23} \mathrm{O}_{2}$ : C. 83.60: H, 6.71: O. 9.68, Found: C. 83.69: H, 6.68 .

cis, exo-2-Styryl-3-(4-methoxyphenylethynyl)bicyclo[2,2.1]heptene (5Ha): ${ }^{1} \mathrm{H}$ NMR $\left(400 \mathrm{MHz}, \mathrm{CDCl}_{3}\right) \delta 1.53\left(>\mathrm{CH}_{2}\right.$ (7). d. $J=8.8 \mathrm{~Hz}, \mathrm{lH}) .1 .98\left(>\mathrm{CH}_{2}(7)\right.$. d. $\left.J=8.8 \mathrm{~Hz}, 1 \mathrm{H}\right)$. $2.46(\mathrm{t}, J=8 \mathrm{~Hz} .1 \mathrm{H}), 2.73(\mathrm{t}, 2 \mathrm{H}), 3.07(\mathrm{~s}, 1 \mathrm{H}) .3 .77$ $\left(-\mathrm{OCH}_{3}\right.$. s. $\left.3 \mathrm{H}\right), 6.18(-\mathrm{CH}=\mathrm{CH}-(5.6)$, s. $1 \mathrm{H}), 6.24(-\mathrm{CH}=$ $\mathrm{CH}-(5.6)$, s. $\mathrm{IH}) .6 .48\left(-\mathrm{C}_{6} \mathrm{H}_{4-}, \mathrm{d}, J=8.8 \mathrm{~Hz} .2 \mathrm{H}\right) .6 .75$ $\left(-\mathrm{C}_{6} \mathrm{H}_{4}-\right.$, d. $\left.J=8.8 \mathrm{~Hz}, 2 \mathrm{H}\right) .7 .18-7.29\left(\mathrm{C}_{6} \mathrm{H}_{-}-\right.$and double bond in styrene. $\mathrm{m}, 5 \mathrm{H}), 7.39\left(\mathrm{C}_{6} \mathrm{H}_{5-}, \mathrm{d}, J=8.8 \mathrm{~Hz}, 2 \mathrm{H}\right)$ ppm; ${ }^{13} \mathrm{C}$ NMR $\left(100 \mathrm{MHz}, \mathrm{CDCl}_{3}\right) \delta 36,44.5,45.6 .48 .8$. $\left.49.9,55.2\left(-\mathrm{C}_{6} \mathrm{H}_{3} \mathrm{OCH}_{3}-\right) .83 .3 .90 .2(-\mathrm{C}=\mathrm{C}-)^{-}\right) .113 .8,116.2$. 126. 1, 126.8. 128.5. 129.8, 132.8, 133.5. 133.7. 137.9. 138. $159 \mathrm{ppm} ;$ IR $(\mathrm{KBr}) 3059.3026,2972.2872,2835.2220$ $(-\mathrm{C}=\mathrm{C}-), 1606,1570(-\mathrm{CH}=\mathrm{CH}-(5,6)), 1510.1450 .1288$. 1248, 1172. 1105. 1070. 1030, 962 (E-double bond). 902 . 831. 796. 769.748.711. $694 \mathrm{~cm}^{-1}$ : MS (FAB) m/2 326.79 (M+) Anal. Calcd for $\mathrm{C}_{34} \mathrm{H}_{3} 3$ O: C. 88.31: H, 6.79: O. 4.90 . Found: C. 88.23 ; H. 6.82 .

cis, exo-2-(4-Chloro)phenyl-3-(4-methoxy phenylethynyl)bicyclo[2,2.1]-heptene (5Ia): ${ }^{1} \mathrm{H}$ NMR (400 $\left.\mathrm{MHz} . \mathrm{CDCl}_{3}\right) \delta$ $1.66\left(>\mathrm{CH}_{2}(7), \mathrm{d} . J=8.8 \mathrm{~Hz} .1 \mathrm{H}\right) .2 .10\left(>\mathrm{CH}_{2}(7)\right.$. d. $J=8.8$ Hz, IH). $2.91($ d. $J=8.8 \mathrm{~Hz} .1 \mathrm{H}), 2.97($ d. $J=8.8 \mathrm{~Hz}, \mathrm{lH})$. $3.10(\mathrm{~d}, J=17.6 \mathrm{~Hz}, 2 \mathrm{H}) .3 .74\left(-\mathrm{OCH}_{\text {s. }} \mathrm{s} .3 \mathrm{H}\right), 6.22(-\mathrm{CH}=$ $\mathrm{CH}-(5,6), \mathrm{s}, \mathrm{IH}), 6.36(-\mathrm{CH}=\mathrm{CH}-(5,6), \mathrm{s}, \mathrm{IH}) .6 .69$ $\left(-\mathrm{C}_{6} \mathrm{H}_{4}-, \mathrm{d} . J=9.6 \mathrm{~Hz}, 2 \mathrm{H}\right), 6.81\left(-\mathrm{C}_{6} \mathrm{H}_{4}-, \mathrm{d} . J=8.8 \mathrm{~Hz}, 2 \mathrm{H}\right)$. $7.17\left(-\mathrm{C}_{6} \mathrm{H}_{4}\right.$, d. $\left.J=8.4 \mathrm{~Hz}, 2 \mathrm{H}\right) .7 .28\left(-\mathrm{C}_{6} \mathrm{H}_{4}, \mathrm{~d}, J=8.8 \mathrm{~Hz}\right.$. $2 \mathrm{H}) \mathrm{ppm}:{ }^{13} \mathrm{C}$ NMR $\left(100 \mathrm{MHz} . \mathrm{CDCl}_{3}\right) \delta 37.2,45.8 .46 .3$. 47.7, 49.7. $55.2\left(-\mathrm{C}_{6} \mathrm{H}_{3} \mathrm{OC} \mathrm{CH}_{3}-\right), 84.4 .90 .4(-\mathrm{C}=\underline{\mathrm{C}}-) .113 .6$. 115.8. 127.8. 130.0. 131.4. 132.6. 136.7. 139.6, 141.6, 158.9 ppm; IR (KBr) 3140, 3063. 2982, 2955. 2934, 29l4. 2837. $2224(-\mathrm{C}=\mathrm{C}-), 2054.1898 .1608,1583.1570(-\mathrm{CH}=\mathrm{CH} \cdot(5$. 6)). $1510.1462,1442.1329,1288,1248,1176,1109.1032$. 910. 837. $810,796.777,767.731 \mathrm{~cm}^{-1}$; MS (EI) $\mathrm{m} / \mathrm{z}$ 334. $1086(\mathrm{M}+)$. Anal. Calcd for $\mathrm{C}_{3} \mathrm{H}_{19} \mathrm{ClO}: \mathrm{C}, 78.91 ; \mathrm{H}$. 5.72: Cl, $10.59 ;$ O. 4.78. Found: C. $79.29 ; \mathrm{H} .5 .83 ; \mathrm{Cl} .10 .52$.

cis, exo-1,4-bis[3-\{2-(4-methoxyphenyl ethynyl) bicyclo[2,2.1]heptenyl\}]-benzene (5Ka): ${ }^{1} \mathrm{H}$ NMR $(400 \mathrm{MHz}$. $\left.\mathrm{CDCl}_{3}\right) \delta 1.68\left(>\mathrm{CH}_{2}(7) . \mathrm{d} . J=8.8 \mathrm{~Hz} .2 \mathrm{H}\right), 2.17\left(>\mathrm{CH}_{2}(7)\right.$. d. $J=8.8 \mathrm{~Hz} .2 \mathrm{H}) \cdot 2.94-3.14(-\mathrm{CH}(\mathrm{l}) . \mathrm{CH}(2), \mathrm{CH}(3)$, and $\mathrm{CH}(4), \mathrm{m} .8 \mathrm{H}), 3.74\left(-\mathrm{OCH}_{3}, \mathrm{~s}, 6 \mathrm{H}\right), 6.23(-\mathrm{CH}=\mathrm{CH}-(5,6)$. s. $2 \mathrm{H}), 6.39(-\mathrm{CH}=\mathrm{CH}-(5.6)$. s. $2 \mathrm{H}), 6.67\left(-\mathrm{C}_{6} \mathrm{H}_{4^{-}}, \mathrm{d} . J=8.8\right.$ $\mathrm{Hz}, 4 \mathrm{H}), 6.79\left(-\mathrm{C}_{6} \mathrm{H}_{4}=\mathrm{d}, J=8.8 \mathrm{~Hz}, 4 \mathrm{H}\right), 7.28\left(-\mathrm{C}_{6} \mathrm{H}_{4}-\mathrm{d} . J\right.$ $=8.8 \mathrm{~Hz}, 4 \mathrm{H}) \mathrm{ppm}:{ }^{13} \mathrm{C}$ NMR $\left(100 \mathrm{MHz} . \mathrm{CDCl}_{3}\right) \delta 37.5$. $46.2,47.2 .48 .4,50.1,55.5\left(-\mathrm{C}_{6} \mathrm{H}_{4} \mathrm{OCCH}_{3}-\right) .84 .5,91.4(-\mathrm{C} \equiv$ C.-. $113.7,116.6,128.5,133.0 .136 .9,140.11 .140 .5,159.0$ ppm; IR (KBr) 3138. 3057, 2980. 2937, 2901.2872, 2837. $2222(-\mathrm{C} \equiv \mathrm{C}-), 2056.1901 .1730,1608.1570(-\mathrm{CH}=\mathrm{CH}-(5$. 6)). $1510.1491,1454.1410,1327.1292,1249,1170.1107$, $1087,1039,1030,1012.904 .839,812.779,765.715 .650$, $540.505 \mathrm{~cm}^{-1}$; MS (EI) $\mathrm{m} / \mathrm{z} 522.2531(\mathrm{M}+)$; Anal. Calcd for
$\mathrm{C}_{38} \mathrm{H}_{34} \mathrm{O}_{2}: \mathrm{C}, 87.32: \mathrm{H}, 6.56 ; \mathrm{O}, 6.12$. Found: $\mathrm{C}, 87.21 ; \mathrm{H}$, 6.51 .

cis exo-2-(2-Thiophenyl)-3-(4-methoxyphenylethynyl)bicyclo[2.2,1] heptene (5La): ${ }^{1} \mathrm{H}$ NMR $\left(400 \mathrm{MHz}, \mathrm{CDCl}_{3}\right) \delta$ $1.68\left(>\mathrm{CH}_{2}(7)\right.$ d d $\left.J=8.8 \mathrm{~Hz}, 1 \mathrm{H}\right) .2 .23\left(\mathrm{CH}_{2}(7), \mathrm{d} . J=\right.$ $9.2 \mathrm{~Hz}, 1 \mathrm{H}$ ). $2.87($ d. $J=8.8 \mathrm{~Hz}, 1 \mathrm{H}) .3 .13($ d. $J=8.8 \mathrm{~Hz}$, $2 \mathrm{H}) .3 .25$ (d. $\mathrm{lH}) \cdot 3.75\left(-\mathrm{OCH}_{3}\right.$. s. $\left.3 \mathrm{H}\right) \cdot 6.2(-\mathrm{CH}=\mathrm{CH} \cdot(5.6)$, s, $\mathrm{lH}), 6.3(-\mathrm{CH}=\mathrm{CH} \cdot(5,6)$. s. $\mathrm{lH}) .6 .70\left(-\mathrm{C}_{6} \mathrm{H}_{4}\right.$, d. $J=8.8$ Hz. $2 \mathrm{H}), 6.9 \mathrm{l}\left(-\mathrm{C}_{4} \mathrm{H}_{2} \mathrm{~S}-, \mathrm{s}, \mathrm{lH}\right) .6 .98\left(-\mathrm{C}_{6} \mathrm{H}_{4}\right.$, d. $J=8.8 \mathrm{~Hz}$. $2 \mathrm{H}) .7 .19\left(-\mathrm{C}_{4} \mathrm{H}_{3} \mathrm{~S}-\right.$, s. IH). $7.25\left(-\mathrm{C}_{4} \mathrm{H}_{3} \mathrm{~S}-\right.$, s. $\left.1 \mathrm{H}\right) \mathrm{ppm} ;{ }^{13} \mathrm{C}$ NMR (I00 MHz. CDCl 3 ) $\delta 37.6,44.2 .45 .9,49.1,50.1 .55 .2$ $\left(-\mathrm{C}_{6} \mathrm{H}_{4} \mathrm{OCH}_{3}-\right), 83.8 .89 .9 \quad(-\underline{\mathrm{C}}=\underline{\mathrm{C}}-), 113.6,116.1,123.2$, $124.8,126.1,132.7,136.7,139.146 .8 .158 .9 \mathrm{ppm} ; \mathrm{IR}(\mathrm{KBr})$ 3063. $2974,2874,2835,2224(-\mathrm{C}=\mathrm{C}-\mathrm{-}), 1606,1570(-\mathrm{CH}=$ CH- $(5,6)), 1510.1462,1440,1413,1367,1325,1288$, 1246. 1172, 1105, 1076, 1039, 902.864, 831. 810, 779. 763 , 723. $694 \mathrm{~cm}^{-1}$. Anal. Calcd for $\mathrm{C}_{34} \mathrm{H}_{18}$ OS: C. 78.39: H. 5.92 : O, 5.22; S, 10.46, Found: C. 78.46: H. 6.14: S. 10.22.

cis exo-2-Phenyl-3-(4-cyanopheny lethynyl)bicyclo[2,2.1]heptene (5Ab): ${ }^{1} \mathrm{H}$ NMR $\left(400 \mathrm{MHz}, \mathrm{CDCl}_{3}\right) \delta 1.70\left(\mathrm{CH}_{2}\right.$ (7), d. $J=8.8 \mathrm{~Hz} . \mathrm{lH}) .2 .11\left(>\mathrm{CH}_{2}(7)\right.$. d. $J=8.8 \mathrm{~Hz}, \mathrm{lH}$ ), $2.94($ d. $J=9.6 \mathrm{~Hz}, 1 \mathrm{H}), 3.06($ d. $J=8.8 \mathrm{~Hz}, 1 \mathrm{H}), 3.15(\mathrm{~d}$, 2H). $6.24(-\mathrm{CH}=\mathrm{CH}-(5.6), \mathrm{s} . \mathrm{lH}), 6.4 \mathrm{l}(-\mathrm{CH}=\mathrm{CH}-(5,6) . \mathrm{s}$, 1H). $6.87\left(-\mathrm{C}_{6} \mathrm{H}_{5}\right.$, d. $\left.J=8.4 \mathrm{~Hz} .2 \mathrm{H}\right) .7 .25\left(-\mathrm{C}_{6} \mathrm{H}_{5} . \mathrm{d} . J=8.4\right.$ Hz. $3 \mathrm{H}) .7 .3 \mathrm{l}\left(-\mathrm{C}_{6} \mathrm{H}_{4}-\mathrm{d}, J=8.4 \mathrm{~Hz} .2 \mathrm{H}\right) .7 .40\left(-\mathrm{C}_{6} \mathrm{H}_{4}-\mathrm{d}, J\right.$ $=8.4 \mathrm{~Hz} .2 \mathrm{H}) \mathrm{ppm} ;{ }^{13} \mathrm{C}$ NMR $\left(100 \mathrm{MHz}, \mathrm{CDCl}_{3}\right) \delta 37.7$. 46.3. 46.4. 48.6. 49.9. 83.4. $97.9(-\mathrm{C}=\mathrm{C}-) .110 .7 .119(-\underline{\mathrm{C}}$ $=\mathrm{N}) .126 .2,128.2,128.9,129.2,131.9,132.1,136.7,140.3$. 142.9 ppm: IR (KBr) 3063, 3024. 2962. 2943, 2920. 2874 . $2224(-\mathrm{C} \equiv \mathrm{N}$ and $-\mathrm{C}=\mathrm{C} \cdot), 1602,1570(-\mathrm{CH}=\mathrm{CH}-(5.6))$, 1545. 1498, 1452, 1406. 1311. 1253, 1178, 1138. 1103, 1076. 1008. 910. $841 \mathrm{~cm}^{-1}$ : MS (FAB) $\mathrm{m} / \mathrm{z} 295.81(\mathrm{M}+$ ); Anal Calcd for $\mathrm{C}_{22} \mathrm{H}_{3} \mathrm{~N}$ : C, 89.46: H. 5.80; N, 4.74 Found: C, $88.61: \mathrm{H}, 5.94 ; \mathrm{N} .4 .51$.

cis, exo-2-(4-Dodecyloxy)phenyl-3-(4-cyanophenylethynyl)bicyclo[2,2.1]-heptene (5.jb): ${ }^{1} \mathrm{H}$ NMR (400 MHz, $\left.\mathrm{CDCl}_{3}\right) \delta$ $0.88\left(-\mathrm{CH}_{3}\right.$, t. $\left.J=6.4 \mathrm{~Hz} .3 \mathrm{H}\right), 1.27-1.43\left(-\mathrm{CH}_{2}=, \mathrm{m}, 18 \mathrm{H}\right)$, $1.69\left(\left(\mathrm{CH}_{2}(7) . \mathrm{d}, \mathrm{lH}\right), 1.76\left(-\mathrm{CH}_{2}, \mathrm{~m} .2 \mathrm{H}\right) .2 .1\left(\left(\mathrm{CH}_{2}(7) . \mathrm{d}\right.\right.\right.$. $J=8 \mathrm{~Hz} . \mathrm{lH}) .2 .9(\mathrm{~d}, J=8.8 \mathrm{~Hz} \cdot \mathrm{lH}) .3 .0(\mathrm{~d}, J=8.8 \mathrm{~Hz}$, lH). $3.1(\mathrm{~d}, J=10.8 \mathrm{~Hz} .2 \mathrm{H}) .3 .94\left(-\mathrm{OCH}_{z-}, \mathrm{t} . J=6.4 \mathrm{~Hz}\right.$. 2H). $6.2 \mathrm{l}(-\mathrm{CH}=\mathrm{CH}-(5.6)$, s. $\mathrm{lH}), 6.39(-\mathrm{CH}=\mathrm{CH}-(5,6) . \mathrm{s}$, 1H). $6.85\left(-\mathrm{C}_{6} \mathrm{H}_{4}-, \mathrm{d}, J=8.4 \mathrm{~Hz}, 2 \mathrm{H}\right) .6 .94\left(-\mathrm{C}_{6} \mathrm{H}_{4}-, \mathrm{d}, J=\right.$ $8.4 \mathrm{~Hz}, 2 \mathrm{H}) .7 .15\left(-\mathrm{C}_{6} \mathrm{H}_{4}-\right.$, d. $\left.J=8.4 \mathrm{~Hz}, 2 \mathrm{H}\right) .7 .41\left(-\mathrm{C}_{6} \mathrm{H}_{4^{-}}\right.$, d. $J=8.4 \mathrm{~Hz} .2 \mathrm{H}) \mathrm{ppm}:{ }^{13} \mathrm{C}$ NMR $\left(100 \mathrm{MHz} . \mathrm{CDCl}_{3}\right) \delta$ 14.1. 22.6, 26.1, 29.3-29.6. 31.9. 37.4, 45.9. 46.5, 47.6. 49.5. 68.1. 83.2. $97.9(-\underline{C} \equiv \underline{C}-), 110.2,113.9 .118 .7 .129 .129 .4$, 131.6. 131.8. 134.4. 136.3, 140.0, 157.4 ppm; IR (KBr) 3061. 2962. 2918. 2849,2224 ( $-\mathrm{C} \equiv \mathrm{C} \cdot), 1602,1581(-\mathrm{CH}=\mathrm{CH}-(5$. 6)), 1545. 1510. 1469, 1394. 1329, 1305. 1292. 1248, 1205. $1176,1140.1107,1024,918,904.842 .810,783 \mathrm{~cm}^{-1}$; MS (FAB) $\mathrm{m} / \mathrm{z} 479.8\left(\mathrm{M}+\right.$ ); Anal. Calcd for $\mathrm{C}_{34} \mathrm{H}_{4} \mathrm{NO}$ : $\mathrm{C}$, 85.13: H. 8.61: N. 2.92: O. 3.34, Found : C, 84.59; H. 8.69; $\mathrm{N}, 2.43$.

cis exo-2-Phenyl-3-pheny lethynylbicyclo[2.2.1] heptene (5Ac): ${ }^{1} \mathrm{H}$ NMR $\left(400 \mathrm{MHz}, \mathrm{CDCl}_{3}\right) \delta 1.67\left(\mathrm{CH}_{2}(7)\right.$, d. $J=$ $8.8 \mathrm{~Hz} . \mathrm{lH}) .2 .16\left(>\mathrm{CH}_{2}(7)\right.$. d. $\left.J=8.8 \mathrm{~Hz}, \mathrm{lH}\right), 2.93(\mathrm{~d} . J=$ $8.8 \mathrm{~Hz} . \mathrm{lH}), 3.02$ (d. $J=8.8 \mathrm{~Hz}, \mathrm{lH}), 3.13($ s. $2 \mathrm{H}), 6.22$ 
$(-\mathrm{CH}=\mathrm{CH}-(5.6)$ s. $\mathrm{lH}), 6.38(-\mathrm{CH}=\mathrm{CH}-(5.6), \mathrm{s}, \mathrm{lH}), 6.85$ $\left(-\mathrm{C}_{6} \mathrm{H}_{5}, \mathrm{~d} . J=8.8 \mathrm{~Hz}, 2 \mathrm{H}\right), 7.12\left(-\mathrm{C}_{6} \mathrm{H}_{5}, \mathrm{~m}, 3 \mathrm{H}\right) .7 .27\left(-\mathrm{C}_{6} \mathrm{H}_{5}\right.$. m, $5 \mathrm{H}) \mathrm{ppm}:{ }^{13} \mathrm{C}$ NMR $\left(100 \mathrm{MHz}, \mathrm{CDCl}_{3}\right) \delta 37.3 .45 .9$. $46.3,48.3 .49 .8,84.2 .92 .4(-\mathrm{C}=\mathrm{C}-), 125.7,128.5 .129 .4$. $131.3,136.6,139.9,1+2.9 \mathrm{ppm} ; \mathrm{IR}(\mathrm{KBr}) 3059.3026 .2974$. $2872,2224(-\mathrm{C} \equiv \mathrm{C}-)$ ) 1946, 1878, 1803. 1726. 1599. 1574 ($\mathrm{CH}=\mathrm{CH} \cdot(5,6)) .1491,1452,1315,1253,1217,1182,1157$. $1099,1070,1028,1012,910,817,792.756,711,692 . \mathrm{cm}^{-1}$. Anal. Calcd for C21H18: C, 93.29; H, 6.71, Found: C. 93.37: H, 6.75.

cis, exo-2-(4-Acetylphenyl)-3-phenylethyny lbicyclo[2,2.1]heptene (5Dc): ${ }^{1} \mathrm{H}$ NMR (400 MHz, $\left.\mathrm{CDCl}_{3}\right) \delta \mathrm{i} .73\left(>\mathrm{CH}_{2}\right.$ (7). d. $J=8.8 \mathrm{~Hz}, \mathrm{lH}) .2 .14\left(>\mathrm{CH}_{2}(7) . \mathrm{d}, J=8.8 \mathrm{~Hz}, \mathrm{lH}\right)$. $2.58\left(-\mathrm{CH}_{3}\right.$, s. $\left.3 \mathrm{H}\right) .2 .99$ (d. $\left.J=8.8 \mathrm{~Hz}, \mathrm{IH}\right) .3 .05$ (d. $J=8.8$ $\mathrm{Hz}, 1 \mathrm{H}), 3.16(\mathrm{~s}, 2 \mathrm{H}), 6.25(-\mathrm{CH}=\mathrm{CH} \cdot(5,6)$, s. $\mathrm{lH}) .6 .39$ $(-\mathrm{CH}=\mathrm{CH}-(5.6) . \mathrm{s}, \mathrm{lH}), 6.80\left(-\mathrm{C}_{6} \mathrm{H} s . \mathrm{d} . J=8.0 \mathrm{~Hz} .2 \mathrm{H}\right)$. $7.12\left(-\mathrm{C}_{6} \mathrm{H}_{5} \mathrm{~m} .3 \mathrm{H}\right), 7.36\left(-\mathrm{C}_{6} \mathrm{H}_{4}, \mathrm{~d}, J=8.4 \mathrm{~Hz}, 2 \mathrm{H}\right), 7.91$ $\left(-\mathrm{C}_{6} \mathrm{H}_{4}-\right.$, d, $\left.J=8.4 \mathrm{~Hz}, 2 \mathrm{H}\right) \mathrm{ppm} ;{ }^{13} \mathrm{C}$ NMR $(100 \mathrm{MHz}$. $\left.\mathrm{CDCl}_{3}\right) \delta 26.6,37.4,45.9,46.1 .48 .5 .49 .8 .84 .5,91.8(-\mathrm{C} \equiv$ C.-. 123.5, 127.4. 127.9. 128.9, 131.1. 134.9. 136.8, 139.6. $149.0,197.9(>\underline{C}=\mathrm{O}) \mathrm{ppm}: \mathrm{IR}(\mathrm{KBr}) 3059.2986 .2972$. $2945,2930,2874,2220(-\mathrm{C} \equiv \mathrm{C}-) .1680 .1604 .1570(-\mathrm{CH}=$ $\mathrm{CH}-(5.6)), 1543,1523,1508,1489,1452,1440,1+13$. $1358,1332,1307.1269 .1186 \mathrm{~cm}^{-1}$ : MS (FAB) $\mathrm{m} / \mathrm{z} 312.15$ $(\mathrm{M}+)$ : Anal. Calcd for $\mathrm{C}_{33} \mathrm{H}_{3 j} \mathrm{O}: \mathrm{C} .88 .43 ; \mathrm{H} .6 .45: 0,5.12$. Found: C. 87.09; H. 6.17.

cis, exo-2-(4-Methylphenyl)-3-phenylethynylbicyclo[2,2.1]heptene (5Ec): ${ }^{1} \mathrm{H}$ NMR $\left(400 \mathrm{MHz} . \mathrm{CDCl}_{3}\right) \delta 1.66\left(\mathrm{CH}_{2}\right.$ (7). d. $J=8.8 \mathrm{~Hz}, \mathrm{lH}) .2 .15\left(>\mathrm{CH}_{2}(7) . \mathrm{d}, J=8.8 \mathrm{~Hz}, \mathrm{lH}\right)$. $2.29\left(-\mathrm{CH}_{\text {. }}\right.$ s. $3 \mathrm{H}$ ). 2.90 (d. $J=8.8 \mathrm{~Hz}, \mathrm{IH}$ ). 2.99 (d. $J=8.8$ $\mathrm{Hz}, \mathrm{IH}) .3 .11(\mathrm{~d} . J=8.0 \mathrm{~Hz}, 2 \mathrm{H}) .6 .2 \mathrm{l}(-\mathrm{CH}=\mathrm{CH}-(5,6) . \mathrm{s}$. $1 \mathrm{H}), 6.37(-\mathrm{CH}=\mathrm{CH}-(5.6), \mathrm{s}, 1 \mathrm{H}), 6.84\left(-\mathrm{C}_{6} \mathrm{H}_{5}, \mathrm{~d}, J=8.0\right.$ $\mathrm{Hz}, 2 \mathrm{H}), 7 . \mathrm{I}+\left(-\mathrm{C}_{6} \mathrm{H}_{4}-\right.$ and $\left.-\mathrm{C}_{6} \mathrm{H}_{5}, \mathrm{~m} .7 \mathrm{H}\right) \mathrm{ppm}:{ }^{13} \mathrm{C}$ NMR $\left(100 \mathrm{MHz}, \mathrm{CDCl}_{3}\right) \delta 20.9,37.2,45.9 .46 .4 .47 .9,49.7,84.2$. $92.6(-\underline{C}=\mathrm{C}-) .123 .9 .127 .1,127.8 .128 .5 .131 .3,135.1$. 136.5. 139.8 ppm; IR (KBr) 3057. 2974. 2945. 2922. 2872. $2224(-\mathrm{C} \equiv \mathrm{C}-), 1599,1572(-\mathrm{CH}=\mathrm{CH}-(5,6)), 1514,1491$. $1450,1377,1329.1315,1253,1219,1155.1111,1068$. $1012.912 .837 .804 .756 \mathrm{~cm}^{-1}$; MS (FAB) $\mathrm{m} / \mathrm{z} 284.16(\mathrm{M}+$ ): Anal. Calcd for $\mathrm{C}_{23} \mathrm{H}_{2 \mathrm{ci}}$ C. 92.91 : H. 7.09. Found: C. 92.00 . H. 6.86 .

$c i s$, exo-2-(4-Methoxyphenyl)-3-phenylethynylbicyclo[2,2.1]heptene (5Fc): ${ }^{~} \mathrm{H}$ NMR $\left(400 \mathrm{MHz} . \mathrm{CDCl}_{3}\right) \delta 1.67\left(\left(\mathrm{CH}_{2}\right.\right.$ (7). d. $J=8.8 \mathrm{~Hz}, \mathrm{lH}) .2 .16\left(>\mathrm{CH}_{2}(7) . \mathrm{d} . J=8.8 \mathrm{~Hz}, \mathrm{lH}\right)$. $2.91($ d. $J=8.8 \mathrm{~Hz} .1 \mathrm{H}) .2 .97(\mathrm{~d} . J=8.8 \mathrm{~Hz} .1 \mathrm{H}) .3 .10(\mathrm{~d} . J$ $=20 \mathrm{~Hz} .2 \mathrm{H}), 3.80\left(-\mathrm{OCH}_{3} \mathrm{~s}, 3 \mathrm{H}\right) .6 .21(-\mathrm{CH}=\mathrm{CH}-(5,6) \mathrm{s}$, $\mathrm{lH}), 6.37(-\mathrm{CH}=\mathrm{CH}-(5,6), \mathrm{s}, \mathrm{lH}), 6.88\left(-\mathrm{C}_{6} \mathrm{H}_{4}-\right.$ and $-\mathrm{C}_{6} \mathrm{H}_{5}$. m. $4 \mathrm{H}), 7.16\left(-\mathrm{C}_{6} \mathrm{H}_{4}\right.$ - and $\left.-\mathrm{C}_{6} \mathrm{H}_{5}, \mathrm{~m} .5 \mathrm{H}\right) \mathrm{ppm} ;{ }^{13} \mathrm{C}$ NMR $\left(100 \mathrm{MHz}, \mathrm{CDCl}_{3}\right) \delta 37.2,45.8,46.6 .47 .6 .49 .7,55.4,84.3$. $92.6 \quad(-\mathrm{C}=\mathrm{C}-), 123.5,113.3 .123 .9 .127 .2,127.8,129.5$. $131.3,134.9 .136 .5,139.9,157.8 \mathrm{ppm}: \mathrm{IR}(\mathrm{KBr}) 3059,2974$. $2872,2833.2224(-\mathrm{C} \equiv \mathrm{C}-), 1612.1583(-\mathrm{CH}=\mathrm{CH}-(5.6))$. $1512,1491,1462,1442,1329,1305,1290,1249,1180$. 1157. 1111. 1068, 1037, 912, 841, 806,785. 756.736. 690 $\mathrm{cm}^{-1}$ : MS (FAB) $\mathrm{m} / \mathrm{z} 300.15(\mathrm{M}+)$; Anal. Calcd for $\mathrm{C}_{2} \mathrm{H}_{3 \mathrm{j}} \mathrm{O}$ : C. 87.96; H. 6.71: O. 5.33, Found: C. 88.04; H. 6.87.

cis, exo-2-Phenyl-3-(4-methoxyphenylethynyl)bicyclo[2,2.1]- heptane (6Ba): ${ }^{1} \mathrm{H}$ NMR $\left(400 \mathrm{MHz} . \mathrm{CDCl}_{3}\right) \delta 1.39\left(>\mathrm{CH}_{2}-\right.$ $\left.\mathrm{CH}_{2}<(5,6), \mathrm{m}, 3 \mathrm{H}\right) .1 .66\left(>\mathrm{CH}_{2}(7)\right.$ and $\mathrm{C}(5)$. s. $\left.2 \mathrm{H}\right) .2 .10$ $\left(>\mathrm{CH}_{2}(7), \mathrm{s}, \mathrm{lH}\right), 2.53$ (s. $\left.\mathrm{lH}\right), 2.59$ (s, $\left.1 \mathrm{H}\right) .3 .04$ (s. $\left.2 \mathrm{H}\right)$. $3.73\left(-\mathrm{OCH}_{3}\right.$. s. $\left.3 \mathrm{H}\right) .6 .64\left(-\mathrm{C}_{6} \mathrm{H}_{4} \cdot\right.$ d. $\left.J=7.2 \mathrm{~Hz} .2 \mathrm{H}\right) .6 .76$ $\left(-\mathrm{C}_{6} \mathrm{H}_{4}=\right.$. m. $\left.2 \mathrm{H}\right) .7 .27\left(-\mathrm{C}_{6} \mathrm{H}_{5} . \mathrm{m} .5 \mathrm{H}\right) \mathrm{ppm} ;{ }^{12} \mathrm{C}$ NMR $(100$ $\left.\mathrm{MHz}_{1} \mathrm{CDCl}_{3}\right) \delta 28.4,31.3 .36 .8,41.5 .43 .2 .44 .4,52.2 .55 .5$. 84.5.90.6 (-C=C-). 113.8, 116.5, 125.9. 128.0. 128.7, 132.9. 143.9. 159.0 ppm: IR (KBr) 3061. 3026. 3003, 2955, 2912. 2868. 2837, 2224 (-C=C.-), 1606. 1508, 1464, 1450. 1413, 1352. 1327, 1294. 1280, 1246, $1197 \mathrm{~cm}^{-1}$; MS (EI) $\mathrm{m} / \mathrm{z}$ 302.1622(M+): Anal. Calcd for $\mathrm{C}_{2} \mathrm{H}_{22} \mathrm{O}: \mathrm{C}, 87.38: \mathrm{H}, 7.33$; O. 5.29. Found: C. 87.09: H. 7.45.

cis. exo-2-(4-Methoxy)phenyl-3-(4-methoxyphenylethynyl)bicyclo[2.2,1]heptane (6Ga): ${ }^{1} \mathrm{H}$ NMR $\left(400 \mathrm{MHz}, \mathrm{CDCl}_{2}\right) \delta$ $1.37\left(>\mathrm{CH}_{2}-\mathrm{CH}_{2}<(5.6) . \mathrm{m}, 3 \mathrm{H}\right) .1 .64\left(>\mathrm{CH}_{2}(7)\right.$ and $\mathrm{C}(5)$, s, $2 \mathrm{H}) .2 .10\left(\mathrm{CH}_{2}(7)\right.$, s. d. $\left.J=6.4 \mathrm{~Hz}, 1 \mathrm{H}\right), 2.52$ (s. $2 \mathrm{H}$ ), 2.99 (m, $2 \mathrm{H}), 3.73$ (s. $3 \mathrm{H}) \cdot 3.79$ (s. $3 \mathrm{H}) .6 .76\left(-\mathrm{C}_{6} \mathrm{H}_{4}-\mathrm{d}, J=\right.$ $8.0 \mathrm{~Hz}, 2 \mathrm{H}) .6 .86\left(-\mathrm{C}_{6} \mathrm{H}_{4}-\right.$ d. $\left.. J=8.4 \mathrm{~Hz}, 2 \mathrm{H}\right) .7 .26\left(-\mathrm{C}_{6} \mathrm{H}_{4}-\right.$ d. $J=8.4 \mathrm{~Hz}, 2 \mathrm{H}) 7.37\left(-\mathrm{C}_{6} \mathrm{H}_{4} \cdot . \mathrm{d} . J=8.4 \mathrm{~Hz} .2 \mathrm{H}\right) \mathrm{ppm}:{ }^{13} \mathrm{C}$ $\operatorname{NMR}\left(100 \mathrm{MHz} . \mathrm{CDCl}_{3}\right) \delta 28.1 .30 .9 .36 .4 .41 .5,42.9,44.1$, 51.1. 55.2, 55.4, 84.2, 90.5 ( $-\mathrm{C}=\underline{\mathrm{C}}-\mathrm{-}) .113 .1,113.5 .116 .2$. 129.3, 132.6, 135.9. 157.6. $158.7 \mathrm{ppm}$; IR (KBr) 3013. 2995 , 2957. $2912,2870.2837 .2226(-\mathrm{C}=\mathrm{C}-), 1606,1583,1510$, 1462. 1440, 1356, 1329, 1290. 1277. 1246. $1176 \mathrm{~cm}^{-1}$; MS (EI) $\mathrm{m} / \mathrm{z} 330.1675(\mathrm{M}+)$ : Anal. Calcd for $\mathrm{C}_{23} \mathrm{H}_{24} \mathrm{O}_{2}: \mathrm{C}$, 83.10: H. 7.28: O, 9.63. Found: C, 82.91; H. 7.36 .

cis, exo-2-(4-Chloro)phenyl-3-(4methoxyphenylethynyl)bicyclo[2.2,1] heptane (6Ia): ${ }^{1} \mathrm{H}$ NMR $\left(400 \mathrm{MHz} \cdot \mathrm{CDCl}_{3}\right) \delta$ $1.37\left(>\mathrm{CH}_{2}-\mathrm{CH}_{2}<(5,6)\right.$. t. $\left.J=11.2 \mathrm{~Hz} .3 \mathrm{H}\right), 1.64\left(>\mathrm{CH}_{2}(7)\right.$ and $\mathrm{C}(5)$. s. $2 \mathrm{H}), 2.05\left(\left(>\mathrm{CH}_{2}\right.\right.$ (7), d. $\left.J=10 \mathrm{~Hz} \mathrm{lH}\right) .2 .52$ (s. lH). 3.01 (q. $J=8.8 \mathrm{~Hz}, 2 \mathrm{H}) .3 .73\left(-\mathrm{OCH}_{3}\right.$, s. $\left.3 \mathrm{H}\right), 6.68$ $\left(-\mathrm{C}_{6} \mathrm{H}_{4}=\mathrm{d} . J=8.8 \mathrm{~Hz} .2 \mathrm{H}\right), 6.79\left(-\mathrm{C}_{6} \mathrm{H}_{4^{-}} \cdot \mathrm{d} . J=8.0 \mathrm{~Hz} .2 \mathrm{H}\right)$, $7.16\left(-\mathrm{C}_{6} \mathrm{H}_{4}-\mathrm{d} . J=8.8 \mathrm{~Hz}, 2 \mathrm{H}\right) .7 .25\left(-\mathrm{C}_{6} \mathrm{H}_{4} \cdot \mathrm{d}, J=8.0 \mathrm{~Hz}\right.$, $2 \mathrm{H}) \mathrm{ppm} ;{ }^{13} \mathrm{C}$ NMR $\left(100 \mathrm{MHz} . \mathrm{CDCl}_{3}\right) \delta 27.9,30.8 .36 .3$. $41.2 .42 .7 .44 .1,51.3,55.2 .84 .6 .89 .9(-C=C \cdot) .113 .6,115.9$. $127.7,129.8,131.3,132.5 .142 .1 .158 .8 \mathrm{ppm}:$ IR $(\mathrm{KBr})$ 3007. $2955,2922.2872,2837.2224(-\mathrm{C}=\mathrm{C}-), 1608.1510$. 1493. 1460, 1412,1329, 1294. 1248. 1194, 1170. 1138. 1105, 1089, 1037, 1012, 987. 956.922, $883.837 \mathrm{~cm}^{-1}$ : MS (EI) $\mathrm{m} / \mathrm{z}$ 336.1226 (M+): Anal. Calcd for $\mathrm{C}_{22} \mathrm{H}_{21} \mathrm{ClO}$ : C, 78.44: H. 6.28: Cl. 10.52; O. 4.75, Found: C, 78.87: H, 6.10; Cl. 11.74 .

\section{References}

1. Kim. J. I.: Lee, J. T.: Choi. C.-K. Bull. Konan Chem Soc. 1986. 7. 235 .

2. Catellani. M.: Chiusoli. G. P. Tetrahedron Lett. 1982. 23.4517.

3. Catellani. M.: Chiusoli. G. P.: Concari. S. Tetrahedron 1989. 45. 5263.

4. Toni, S.: Okumoto. H.; Ozaki, H.: Nakayasu, S.: Kotani, T. Tetahedron Lett. 1990. 31. 5319.

5. Chatani. N.: Amishiro. N.: Murai. S. J. Am. Chem. Soc. 1991. 113. 7778 .

6. Ozawa. F.: Kobatake. Y: Kubo. A.: Hayashi. T. J. Chem. Soc. Chen Conmum. 1994. 1323.

7. Chatani. N:- Amishiro. N.: Morii. T: Yamasita, T.: Murai. S. $d$. Org. Chem. 1995. 60, 1834.

8. (a) Mivaki. N.: Tomita. I.: Endo, T. Hacromolecules 1996, 29 
6685. (b) Miyaki. N.: Tonita I: Endo. T. J. Polm. Sci.: Part A: Potw Chent 1997, 35. 1211. (c) Miyaki. N.: Tomita. I.: Endo. T. J. Polm Sci: Part A: Polm Chem 1997, 35. 2097. (d) Miyaki. N.: Tomita. I.: Endo. T. Macronolecules 1997, 30. 4504. (e) Mivaki. N.: Tomita, I.: Endo, T. Chem. Lett. 1997. 685. (f) Miyaki. N.: Tomita. I.: Endo. T. Pohm. Bull. 1997, 39.677.

9. Kosugi M: Tamura. H.: Sano, H Migita. T. Chem. Lett 1987. 193.

10. Kosugi. M.: Tamura. H.: Sano. H.: Migita. T. Tetrahedron 1989. 45.961 .

11. Kosugi, M.: Kinnura. T.: Oda. H.: Migita, T. Bull Chem. Soc. Jpm. 1993, 66, 3522

12. Oda, H.: Ito. K: Kosugi, M.: Migita, T. Chem. Leth 1994. 1443.

13. Oda. H.: Hamataka. K.: Fugami. K: Kosugi. M.: Migita. T. Sinten 1995. 1225

14. Larock. R. C.: Babu. S. Tetrahedron 1987, 43. 2013.

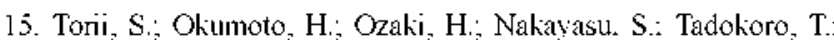
Kotani. T. Tetrahedron Lett. 1992. 33. 3499.

16. Torii. S.: Okumoto. H.: Kotani. H. T.: Nakavasu. S.: Ozaki. H. Tetrahedron Lett. 1992. 33. 3503.

17. Both bis(tributylstannyl)acetylene and 2.5-dimethyl-3-hexyn-2.5diol are available from Aldrich Co. Ine, whose prices are $\$ 8,500$ : mole and S31imole, respectively derived from the largest sized bottles listed in the $1996-97$ catalog.

18. (a) De Mora. S. I. Tributhin Case Stuty of an Entromental Contaminamt. Cambridge University: Cambridge. 1966. (b) Bover. I. J. Toxicology 1989, 55,253 .

19. (a) Huynh. C.: Linstrumelle. G. Tetrahedron 1988, 41. 6337. (b) Solomin. V. A.: Heitz, W. Hacromol. Chem. Phns. 1994, 195. 303

20. (a) The Organic Chentistry of Palladimn: Maitlis. P. M.. Ed.: Academic: New York. 1971: Vol. II 